\section{Gradual initiation of dialysis as a means to reduce cost while providing quality health care}

\section{Mohamed E. Elrggal and Rowan Zyada}

We read with interest the comprehensive Review by R. Vanholder et al. (Reducing the costs of chronic kidney disease while delivering quality health care: a call to action. Nat. Rev. Nephrol. 13, 393-409; 2017) $)^{1}$. In their Review, the authors provide an excellent discussion of the cost of care for patients with chronic kidney disease (CKD) and end-stage renal disease (ESRD) as well as approaches to decrease these costs without affecting the quality of care. We would, however, like to add to the list of proposed approaches to decrease the costs associated with renal replacement therapy, the concept of incremental haemodialysis, whereby patients undergo a gradual transformation from conservative CKD care to dialysis. Although thrice weekly haemodialysis is the standard of care in most parts of the world, patients with ESRD continue to experience low health-related quality of life', accelerated loss of residual kidney function (RKF) ${ }^{3}$ and high mortality rates, especially in the first year of dialysis initiation ${ }^{4,5}$. Moreover, dialysis places a huge financial burden on health-care systems.

Gradual initiation of haemodialysis could provide a more individualized approach for patients with incident ESRD, with the frequency of dialysis determined by various criteria, including residual urine volume (RUV), RKF, cardiovascular status (that is, the presence or absence of heart failure), the presence of comorbidities, findings from pre-dialysis laboratory investigations (for example, acidosis and levels of potassium, phosphorus, haemoglobin, and albumin), nutritional status and self-motivation. For example, once weekly dialysis sessions could facilitate the smooth initiation of dialysis for certain individuals without negatively affecting their social activities, working hours and, theoretically through preservation of $\mathrm{RKF}^{6}$ and cardiovascular condition (reported to be the main cause of mortality in the first year of dialysis initiation $\left.{ }^{5,7,8}\right)$. Such an incremental approach to dialysis initiation also allows additional time to plan for fistula creation and maturation, preserves RKF and decreases health-care costs ${ }^{9}$. Cost reductions would not only result from the reduced number of haemodialysis sessions, but would also ensue due to vascular access preservation owing to the lower frequencies of punctures, reduced hospitalization from myocardial ischaemic events (as preserved $\mathrm{RKF}^{6}$ and RUV enables a lower ultrafiltration rate, which is associated with a low risk of myocardial stunning $)^{9}$ and reduced absence from work.

Approaches to introduce such a strategy, however, face two main problems. The first involves teaching nephrologists to identify patients who might benefit from incremental haemodialysis ${ }^{2}$. The second issue relates to approaches to monitor RKF and when to increase dialysis frequency. We suggest that RKF monitoring could be simplified through the use of daily RUV ${ }^{10}$, instead of more cumbersome methods such as $24 \mathrm{~h}$ urea or creatinine clearance. Implementation of incremental haemodialysis will eventually cut the cost of dialysis, at least in the first year of dialysis initiation.

Mohamed E. Elrggal and Rowan Zyada are at the Nephrology Department, Kidney and Urology Center, 236 Porsaid Street, Cleopatra, Alexandria, Egypt. melrgal@hotmail.com; rawanzyada@gmail.com doi:10.1038/nrneph.2017.135 Published online 25 Sep 2017

1. Vanholder, R. et al. Reducing the costs of chronic kidney disease while delivering quality health care: call to action. Nat. Rev. Nephrol. 13, 393-409 (2017)

2. Kalantar-Zadeh, K. et al. Twice-weekly and incremental hemodialysis treatment for initiation of kidney replacement therapy. Am. J. Kidney Dis. 64, 181-186 (2014).

3. Diao, Z. et al. Preservation of residual renal function with limited water removal in hemodialysis patients. Ren. Fail. 33, 875-877 (2011).

4. Zhang, H. et al. Dialysis outcomes and analysis of practice patterns suggests the dialysis schedule affects day-of-week mortality. Kidney Int. 81, 1108-1115 (2012).

5. Bradbury, B. D. et al. Predictors of early mortality among incident US hemodialysis patients in the Dialysis Outcomes and Practice Patterns Study (DOPPS). Clin. J. Am. Soc. Nephrol. 2, 89-99 (2007).

6. Lin, Y.-F. et al. Comparison of residual renal function in patients undergoing twice-weekly versus three-timesweekly haemodialysis. Nephrology (Carlton) 14 59-64 (2009).

7. Eckardt, K.-U. et al. High cardiovascular event rates occur within the first weeks of starting hemodialysis. Kidney Int. 88, 1117-1125 (2015).

8. Steenkamp, R., Rao, A. \& Fraser, S. UK Renal Registry 18 th annual report (December 2015) chapter 5: survival and causes of death in UK adult patients on renal replacement therapy in 2014: national and centre-specific analyses. Nephron 132 (Suppl. 1), 111-144 (2016).

9. Caria, S., Cupisti, A., Sau, G. \& Bolasco, P. The incremental treatment of ESRD: a low-protein diet combined with weekly hemodialysis may be beneficial for selected patients. BMC Nephrol. 15, 172 (2014).

10. Lee, M. J. et al. Prognostic value of residual urine volume, GFR by 24-hour urine collection, and eGFR in patients receiving dialysis. Clin. J. Am. Soc. Nephrol. 12, 426-434 (2017).

Competing interests statement

The authors declare no competing interests. 